\title{
Gastroenteropancreatic neuroendocrine tumours: the current incidence and staging based on the WHO and European Neuroendocrine Tumour Society classification: an analysis based on prospectively collected parameters
}

\author{
Martin B Niederle, Monika Hackl ${ }^{1}$, Klaus Kaserer ${ }^{2}$ and Bruno Niederle \\ Division of General Surgery, Section of Endocrine Surgery, Department of Surgery, Medical University of Vienna, Währinger Gürtel \\ 18-20, A-1090 Vienna, Austria \\ 'Bundesanstalt 'Statistik Österreich' (Austria Statistics), Guglgasse 13, A-1110 Vienna, Austria \\ ${ }^{2}$ Department of Clinical Pathology, Medical University of Vienna, Währinger Gürtel 18-20, A-1090 Vienna, Austria \\ (Correspondence should be addressed to M B Niederle; Email: martin.niederle@meduniwien.ac.at)
}

\begin{abstract}
As incidence data on gastroenteropancreatic neuroendocrine tumours (GEP-NETs) have so far only been retrospectively obtained and based on inhomogeneous material, we conducted a prospective study in Austria collecting all newly diagnosed GEP-NETs during 1 year. Using the current WHO classification, the tumor, nodes, metastases (TNM) staging and Ki67 grading and the standard diagnostic procedure proposed by the European Neuroendocrine Tumor Society (ENETS), GEP-NETs from 285 patients (male: 148; female: 137) were recorded. The annual incidence rates were 2.51 per 100000 inhabitants for men, 2.36 per 100000 for women. The stomach (23\%) was the main site, followed by appendix (21\%), small intestine (15\%) and rectum (14\%). Patients with appendiceal tumours were significantly younger than patients with tumours in any other site. About $46.0 \%$ were classified as benign, $15.4 \%$ as uncertain, $31.9 \%$ as well differentiated malignant and $6.7 \%$ as poorly differentiated malignant. Patients with benign or uncertain tumours were significantly younger than patients with malignant tumours. Among the malignant tumours of the digestive tract, $1.49 \%$ arose from neuroendocrine cells. For malignant gastrointestinal NETs, the incidence was 0.80 per 100 000: 40.9\% were ENETS stage I, $23.8 \%$ stage II, $11.6 \%$ stage III and $23.8 \%$ stage IV. The majority (59.7\%) were grade $1,31.2 \%$ grade 2 and $9.1 \%$ grade 3 . NETs of the digestive tract are more common than previously reported; the majority show benign behaviour, are located in the stomach and are well differentiated. G3 tumours are very rare.
\end{abstract}

Endocrine-Related Cancer (2010) 17 909-918

\section{Introduction}

Neuroendocrine tumours (NETs) of the gastrointestinal tract are rare neoplasms and represent a heterogeneous group of tumours with distinct functional and biological behaviour depending on location, tumour size and clinical symptoms (Kloppel et al. 2004). NETs arise from the neuroendocrine cells of the diffuse neuroendocrine system. Located in the oesophagus, stomach, duodenum, pancreas, ileum, jejunum, appendix, colon or rectum, these tumours are summarised as gastroenteropancreatic (GEP) NETs (Kloppel et al. 2004); they were formerly referred to as gastrointestinal carcinoids, a term introduced by Oberndorfer more than 100 years ago (Kloppel 2007a, Modlin et al. 2007).

NETs were recently classified according to standardised histopathological criteria established by the WHO (Solcia et al. 2000). Irrespective of the site of origin, these endocrine tumours are classified in general 
as 'well differentiated with benign' or 'uncertain', 'well differentiated with low-grade malignant' or 'poorly differentiated with high-grade malignant behaviour'. The classification is based on particular criteria (size, invasion, Ki67 index, etc.) and used to predict a tumour's biological behaviour (benign, malignant) with high probability (Kloppel et al. 2004).

Incidence data on GEP-NETs are difficult to obtain and are mainly based on national cancer registries. These usually document malignant diseases on the basis of clinical reports, and therefore accurate and relevant analysis of the incidence of GEP-NETs may have substantial limitations since the majority of NETs with benign or uncertain clinical course may not be fully incorporated. In addition, such data have usually been collected over an extended period, during which time the definitions of the disease have evolved. Furthermore, information on the incidence of NETs in different sites is not as precisely documented in the national registries as information on adenocarcinoma of the digestive tract.

To our knowledge, the rare papers analysing the incidence of NETs are either retrospective database analyses (Godwin 1975, Hemminki \& Li 2001, Taal \& Visser 2004) or autopsy studies (Berge \& Linell 1976), and they lack both the recently recommended standardised histopathological characterisation and the current WHO classification of NETs.

The aim of this study was to evaluate the incidence of GEP-NETs in a Middle European country (Austria) based on prospectively collected pathological reports, using a standardised histopathological protocol for initial diagnosis. The current WHO recommendations were used for classification (Kloppel et al. 2004), and the recently defined criteria of the European Neuroendocrine Tumour Society (ENETS) were used for staging and grading (Rindi et al. 2006, 2007). In addition, the incidence of malignant GEP-NETs was compared with that of other malignant tumours identified in the same parts of the digestive tract.

\section{Methods}

The objective of the prospective trial was to collect pathology reports of all tumours diagnosed and classified histologically/immunohistochemically as NETs during 1 year (01/05/2004-30/04/2005) to analyse current data on the Austrian national incidence of GEP-NETs.

\section{Incidence}

There are 41 registered departments and institutes of clinical pathology linked to public and private hospitals in Austria. All were invited, and 40 participated in this prospective 1-year study.
With regard to the study protocol, only patients with NETs located in the gastrointestinal tract and pancreas initially diagnosed within the study period were included in the analysis. Of the 40 participating pathological institutes, 31 reported NETs during the study period.

Tumour definition was initially based on typical morphological characteristics (Soga 1997). However, the detailed neuroendocrine origin of the tumours was definitively identified using staining for markers of neuroendocrine differentiation, chromogranin A and/or synaptophysin, as described recently (Kloppel et al. 2004). Neuron-specific enolase was not used for characterisation as it is insufficiently reliable (Kloppel 2007b).

Apart from the histopathological characteristics, additional parameters such as tumour location and the patient's sex and age were documented by the participating institutes, and the pathology reports were sent online to the study centre.

\section{WHO tumour classification}

As published recently (Kloppel et al. 2004), the detected NETs were subdivided, irrespective of the site of the primary tumour, as 'well differentiated' tumours with either 'benign' or 'uncertain' behaviour or as 'well differentiated' or 'poorly differentiated' neuroendocrine carcinomas.

Neuroendocrine liver tumours were included in the analysis if no other neuroendocrine malignancy outside the abdomen (e.g. lung, ovary) was detected at the time of diagnosis and were therefore assessed as distant metastasis from GEP-NETs. For further evaluation of liver tumours, particularly to gain information on location of the primary tumour in the ileum or jejunum, and if adequate amounts of samples were available, tissue was stained immunohistochemically with antibodies against serotonin (Kloppel et al. 2009).

The pathological data were supervised by one of the authors $(\mathrm{K} \mathrm{K})$, and if questionable, additional immunohistochemical studies were conducted.

\section{TNM staging and grading based on ENETS definitions}

Based on the clinical, surgical and imaging data, the recently published TNM classification and staging system, together with the tumour grading suggested by Rindi et al. $(2006,2007)$, was used whenever possible.

\section{Statistical analysis}

A Filemaker database for documentation of patients with NETs was built up, recording all pathological and available clinical parameters. SPSS version 17.0 (Chicago, IL, USA) was used for statistical analyses. 
Data are described as median and interquartile range (IQR). Age-standardised (=age-adjusted) incidence rates were calculated using Austrian population data published by Statistics Austria. The reference population was based on the World standard population, as used by the International Agency for Research on Cancer (Parkin et al. 2003) and modified by Doll et al. (1966) after Segi et al. (1960).

For comparison of the Austrian incidence data to those of the usually cited 'Surveillance, Epidemiology, and End Results' (SEER) Program of the National Cancer Institute from the USA (Modlin et al. 2003, Hauso et al. 2008, Yao et al. 2008), the age-adjusted incidence rate was further calculated to the 2000 US standard population (Klein \& Schoenborn 2001).

The population-based Austrian cancer registry, which recorded all digestive tract cancers in 2004, was used to compare the incidence of malignant NETs with that of gastrointestinal cancer of various sites of the digestive tract. Austria, in central Europe, has about 8.3 million inhabitants, $10 \%$ of whom are foreigners mainly from other countries of the European Union, the former Yugoslavia and Turkey.

$P$ values were calculated using Mann-Whitney $U$ tests and corrected for multiple testing using the Bonferroni-Holm method. $P$ values $<0.05$ were considered significant.

\section{Informed consent}

All the patients were asked to give informed consent for documentation of their pathological and clinical data. The full names of the patients remained unknown to the study centre.

All the patients agreed to participate in the 'pathological analysis', but not all agreed to participate in the 'clinical' part of the study; thus not all patients could be included. Information on the presence or absence of metastasis had to be available as a minimum requirement for ENETS staging.

The design of the prospective study, the manner of data collection and the retrospective analysis were approved by the ethics committee of the Medical University of Vienna (Resolution number 157/2005).

\section{Results}

\section{Incidence}

A total of 305 tumours were reported during the 12-month period. Eleven specimens were excluded because the immunohistochemistry was either negative or impossible to assess because of inadequate material; an additional five were excluded because the date of diagnosis was not within the study period; a further three specimens were metastasis in the liver from NETs in other sites (lung: 2, ovary: 1), and one biopsy could not be related to an organ (biopsy of a NET of the abdomen). Thus, 285 NETs located in the gastrointestinal tract were selected from the database and were made available for detailed evaluation.

\section{Incidence and biological behaviour of NETs}

Adjusted to the World standard population, the overall incidence was 2.39 per 100000 inhabitants per year: 2.36 per 100000 for women and 2.51 per 100000 for men. Calculated to the 2000 US standard population, the overall incidence was 2.99 per 100000 inhabitants per year.

The age-specific incidence rate was highest between 50 and 70 years and began to increase at about 35 years.

Classification of the NETs according to the WHO criteria found that the overall incidence of tumours with benign, uncertain and malignant behaviour was 1.15 , 0.43 and 0.81 per 100000 inhabitants respectively.

\section{Age and sex of patients}

Of the 285 patients, 148 were men and 137 were women; their median ages at initial diagnosis were 63 (IQR: 22) and 59 (IQR: 27) years respectively ( $P$ value significant only before correction for multiple testing: $P<0.05$, then non-significant). Patients with appendiceal NETs were significantly younger (median 38 (IQR: 45) years) than patients with NETs in any other site $(P<0.05)$.

\section{Location}

The site of the primary tumour was the stomach in 65 $(22.8 \%)$ patients, followed by the appendix $(n=59$; $20.7 \%$ ), small intestine (excl. duodenum; $n=44 ; 15.4 \%$ ), rectum $(n=40 ; 15.4 \%)$, pancreas $(n=33 ; 11.6 \%)$, colon $(n=20 ; 7.0 \%)$ and duodenum $(n=16 ; 5.6 \%)$. In one patient, the tumour was located in the oesophagus, and in another patient, it was located in the gallbladder and Meckel's diverticulum (Fig. 1; for analysis of the clinical data in Part 2, these three tumours were excluded).

Liver tumours expressing neuroendocrine markers were documented in five $(1.7 \%)$ patients. By definition (see above), these tumours were categorised as metastasis from NETs located in various sites of the gastrointestinal tract. Adequate amounts of tissue were available for further immunohistochemical studies in three of the tumours: all three stained negative for serotonin. The definitive location of the primary tumour remained unknown during the follow-up period of 1 year. These five tumours were excluded from further clinical analysis. 


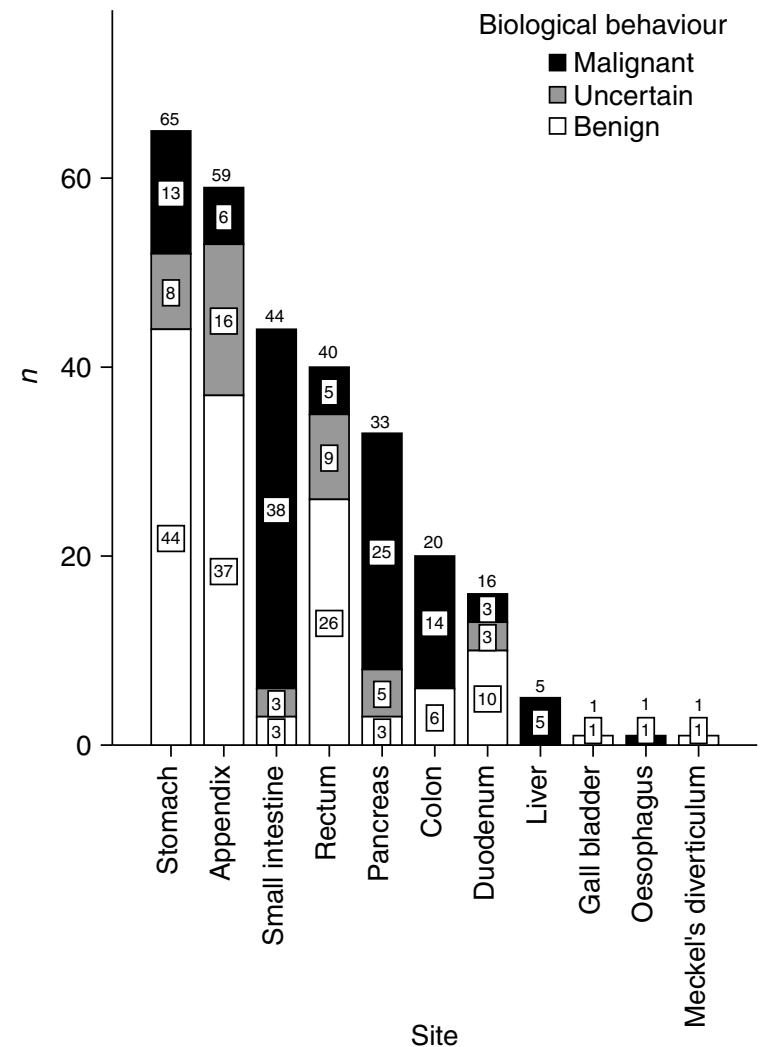

Figure 1 Number, location and biological behaviour of neuroendocrine tumours in each site.

\section{Differentiation and biological behaviour of NETs}

By definition (Kloppel et al. 2004), 131 (46.0\%) NETs were classified as benign (incidence rate 1.15 per $100000), 44(15.4 \%)$ as having uncertain behaviour (incidence rate 0.43 ) and $110(38.6 \%)$ as malignant (incidence rate 0.81). Among the malignant tumours, $91(82.7 \%$; $31.9 \%$ of all 285 tumours) were well differentiated and $19(17.3 \%$; $6.7 \%$ of all 285 tumours $)$ were poorly differentiated.

\section{Age/sex of patients and biological behaviour of tumours}

The median age of patients with benign tumours was 60.0 (IQR: 26) years, with tumours of uncertain behaviour was 51.0 (IQR: 37) years and with malignant tumours was 65.5 (IQR: 18) years. Patients with benign or uncertain tumours were significantly younger than patients with malignant tumours (both $P<0.01$ ); the ages of patients with benign and uncertain tumours were not significantly different from each other.

Even after excluding appendix NETs (patients with appendix NETs were significantly younger; $62.7 \%$ classified benign), patients with benign tumours were younger than patients with malignant tumours $(P<0.05)$ (uncertain versus malignant and benign versus uncertain: $P$ not significant). Among patients with malignant tumours, the median age of those with well differentiated tumours was 60.0 (IQR: 24) years and of those with poorly differentiated tumours was 73.0 (IQR: 17) years $(P<0.05$; after correction for multiple testing $P$ not significant, 0.06).

The distribution of tumours with benign, uncertain and malignant behaviour was approximately equal in male and female patients for all tumour sites.

\section{Location and biological behaviour of tumours}

Whereas NETs in the stomach $(67.7 \%)$, rectum $(65 \%)$ and appendix $(62.7 \%)$ were mainly classified as benign, those in the small intestine (86.4\%), pancreas (75.8\%) and colon $(70.0 \%)$ were predominantly malignant (Fig. 1).

Concerning tumour location, Fig. 2 summarises the malignant NETs after subdividing them into well differentiated and poorly differentiated. Four of five malignant rectal NETs were classified as poorly differentiated, whereas the proportions of this tumour type in all other sites except the duodenum were closely similar. Among pancreatic NETs (PNETs), 20 (80.0\%) of 25 were classified as well differentiated and five as poorly differentiated endocrine carcinoma. Five of five malignant duodenal NETs were classified as well differentiated.

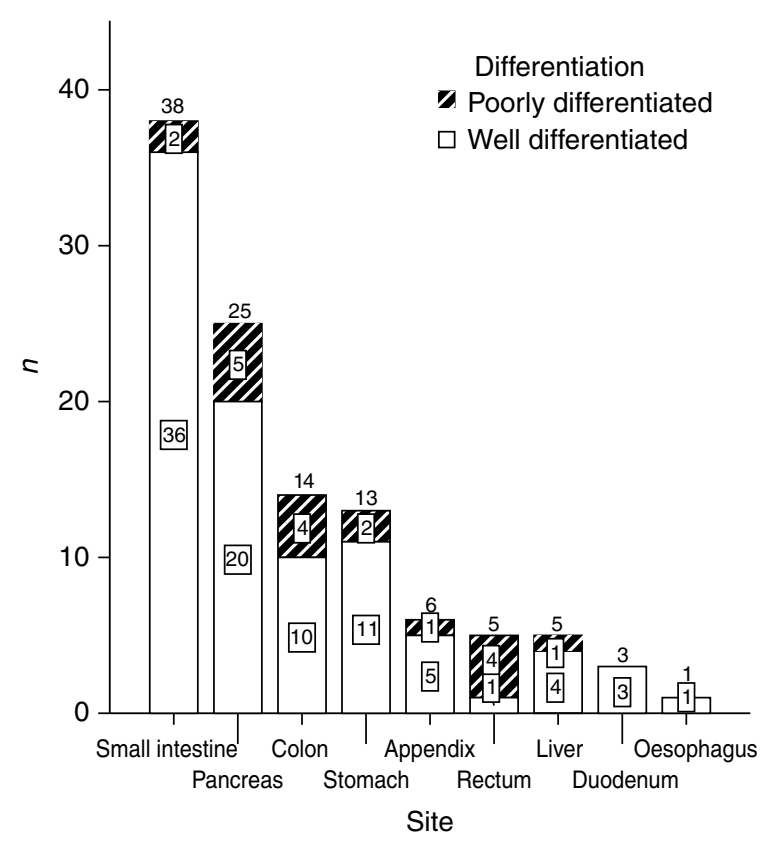

Figure 2 Malignant neuroendocrine tumours of the digestive tract - well differentiated and poorly differentiated neuroendocrine carcinomas. 
Table 1 Site and incidence of malignant neuroendocrine tumours and other malignancies of the digestive tract

\begin{tabular}{|c|c|c|c|}
\hline \multirow[b]{2}{*}{ Site } & \multicolumn{2}{|c|}{ Incidence in digestive tract (per 100000 population) } & \multirow{2}{*}{$\begin{array}{l}\text { Digestive malignant } \\
\text { NETs: percentage of } \\
\text { all malignancies }\end{array}$} \\
\hline & Malignant NETs ${ }^{a}$ & $\begin{array}{l}\text { All reported } \\
\text { malignancies }^{\mathbf{b}}\end{array}$ & \\
\hline Oesophagus & 0.01 & 2.55 & 0.39 \\
\hline Stomach & 0.08 & 8.16 & 0.98 \\
\hline Duodenum & 0.02 & 0.23 & 8.70 \\
\hline Pancreas & 0.19 & 8.31 & 2.29 \\
\hline Small intestine excl. duodenum & 0.29 & 0.56 & 51.79 \\
\hline Appendix & 0.08 & 0.27 & 29.63 \\
\hline Colon (incl. rectosigmoid junction) & 0.06 & 18.19 & 0.33 \\
\hline Rectum & 0.03 & 9.73 & 0.31 \\
\hline Liver & 0.05 & 5.48 & 0.91 \\
\hline Meckel's diverticulum & 0.00 & 0.01 & 0.00 \\
\hline Gall bladder incl. bile duct & 0.00 & 1.00 & 0.00 \\
\hline Total & 0.81 & 54.49 & 1.49 \\
\hline
\end{tabular}

aStudy period: 05/2004-04/2005.

b2004: reference year.

\section{Incidence of malignant NETs and other malignancies in the digestive tract}

Table 1 summarises the incidence of malignant NETs and all reported malignancies in the digestive tract. By definition, $1.49 \%$ of all malignant tumours of the digestive tract documented in the study period arose from neuroendocrine cells. In addition, $51.79 \%$ of all malignant tumours of the small intestine, $29.63 \%$ of all carcinomas of the appendix and $8.70 \%$ of duodenal malignancies were of neuroendocrine origin. The incidence of malignant gastrointestinal NETs was 0.81 per 100000 inhabitants, and the incidence of all reported malignancies in the digestive tract was 54.49 per 100000 inhabitants.

\section{Distant metastasis}

A total of 110 (38.6\%) of 285 tumours were classified as malignant using the WHO criteria. At the time of diagnosis, information on metastasis was available in 87 (79.1\%) patients. Lymph node and distant metastases were documented in $65(74.7 \%)$ of 87 patients. The majority of distant metastases were located in the liver ( $n=35$ : multiple 32, solitary 3 ), followed by the peritoneum $(n=11)$, bone $(n=4)$, lung $(n=2)$, ovary $(n=2)$ and brain $(n=1)$. Extended lymph node and distant metastases were documented in 29 (33.3\%) patients.

\section{TNM staging based on ENETS definitions}

ENETS TNM classification and staging was possible in $181(63.5 \%)$ of 277 tumours. Localised disease (lymph node negative) was documented in $118(65.2 \%)$ of 181 tumours, regional disease (lymph node positive) in 20 $(11.0 \%)$ and distant disease in $43(23.8 \%)$ tumours respectively. Applying the ENETS criteria, 74 tumours $(46.4 \%)$ were stage I, $43(23.8 \%)$ were stage II, 21 $(11.6 \%)$ were stage III and $43(23.8 \%)$ were stage IV independent of their location. Details are summarised in Table 2.

\section{Histopathological grading based on ENETS criteria}

Sufficient data were available in 77 (46.8\%) of 277 tumours. The majority $(46 ; 59.7 \%)$ of the tumours were grade 1, $24(31.2 \%)$ were grade 2 and $7(9.1 \%)$ were grade 3 . Of the seven tumours classified grade 3 , one was located in the stomach, two in the pancreas, two in the colon and two in the rectum.

\section{Discussion}

This is the first study to analyse the incidence of GEPNETs using a prospectively run database. During the 1-year period, 285 tumours were recorded, resulting in an overall annual age-adjusted incidence rate (world standardised) of digestive NETs being 2.39 per 100000 inhabitants per year. The results of the study, in which all locations of the digestive tract were included, show a higher incidence of GEP-NETs than that in other studies analysing similar populations (Helland et al. 2006, Ito et al. 2010).

It may be assumed that nearly all newly diagnosed Austrian cases were recorded within the study period: 40 of 41 departments and institutes of clinical pathology, including four academic institutes, distributed throughout the country participated in the prospective study protocol, and 31 institutes reported tumours diagnosed within the study period. This enables the analysis to attain a high degree of completeness. 


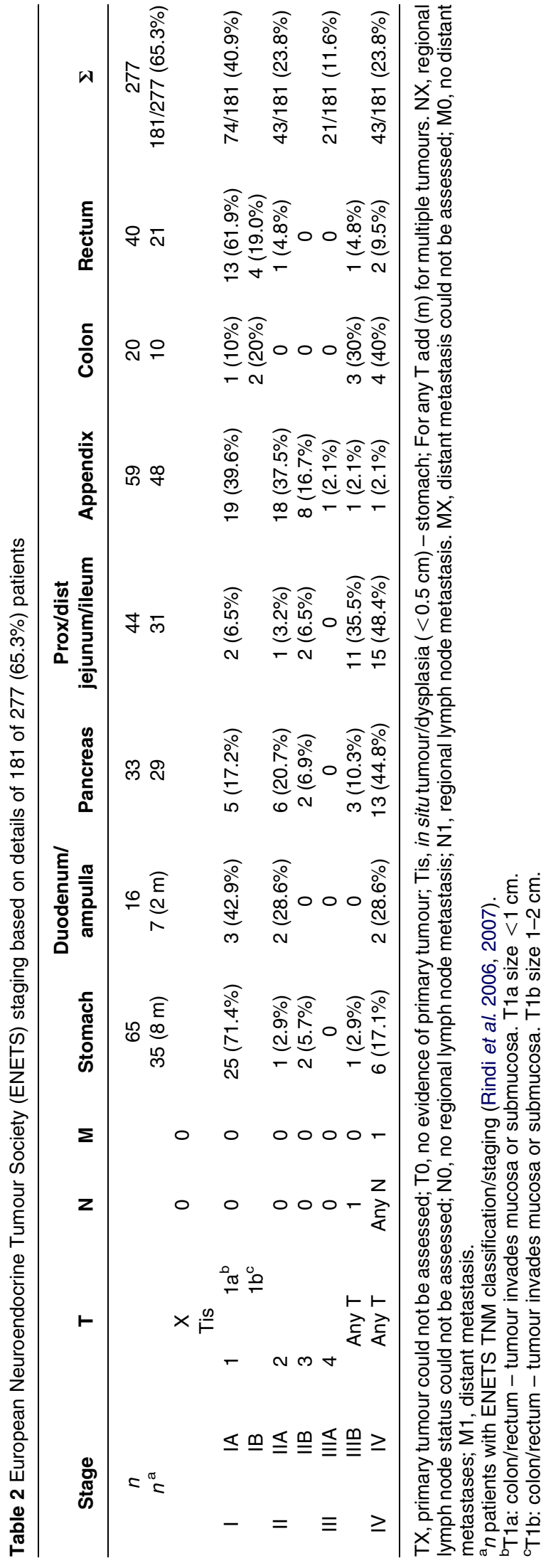

The number of patients diagnosed with GEP-NETs, on the basis of standardised histopathological criteria, and the incidence of these tumours are therefore representative for a central European country with a highly developed medical training and social framework.

Because of vague clinical presentation sometimes and low awareness among physicians, data in the literature on the real incidence of NETs are probably underestimates. Data comparisons in relation to the site of tumour origin are difficult to obtain because of the overall rarity of these neoplasms and their incomplete recognition and documentation in various national cancer registries (Gatta et al. 2006) and because of their variable and sometimes imprecise classification (Kloppel et al. 2009, Klimstra et al. 2010).

NETs were not well-defined entities before 2000 (Solcia et al. 2000), and national tumour databases offer population-based prospective documentation for malignant tumours only. The majority of these databases therefore underreport or even omit NETs classified as benign according to the WHO criteria (Newton et al. 1994, Crocetti et al. 1997) and usually correspond to grade 1 NETs according to the ENETS criteria (Rindi et al. 2006).

Recently, Hauso et al. (2008) and Yao et al. (2008) have reported a worldwide increasing incidence of NETs over the past three decades, suggesting that NETs are more prevalent than previously realised. These findings may partially reflect the more precise pathohistological definition of the neuroendocrine cells with the inclusion of those classified as benign in incidence studies. As shown in our study, NETs classified as benign are the largest group (46.0\%). The higher incidence may also be associated with the incidental identification of small, asymptomatic lesions as the result of increased availability of endoscopic imaging of the upper and lower gastrointestinal tract and the broad use of early radiological imaging in addition to clinical examinations.

In discussion of the incidence of NETs, data from the SEER Program of the National Cancer Institute are usually cited (Modlin et al. 2003, Hauso et al. 2008, Yao et al. 2008). The SEER registries may underestimate the total number of patients with NETs in various locations because all cases from the SEER database are by definition denoted to be malignant. It is therefore likely that small tumours with benign appearance may not generally be included in the SEER registries. One also has to keep in mind that all studies, including the SEER database, lack a standardised histopathological protocol for diagnosis.

In analysis of the late SEER period (1992-1999), Modlin et al. (2003) presented incidence rates of 1.89 
per 100000 for White males and 1.59 per 100000 for White females. Because of higher incidence rates in Black males and females, the number for the White population may be best compared to European population. More recently, Hauso et al. (2008) have compared the SEER NET data with the corresponding data of the Norwegian Registry of Cancer. The ageadjusted incidence rates extracted from 1993 to 2004 using the 2000 US standard population as reference for both databases calculated an incidence rate of 1.99 per 100000 per year for Norway and of 2.3 for the USA (White subset of the SEER data; excluded lung/bronchus, urogenital tract, breast). The same calculation for Austria would show a higher incidence (2.99 per 100000 per year; Fig. 3a) including all GEPNETs independent of their presumed biological behaviour. This 'statistical game' underlines the importance of adjusting incidence calculations to the same (and representative) 'reference population'.

Using the 'World standard population' as reference, Hemminki \& Li (2001) calculated incidence rates of 1.6 per 100000 for males and 1.9 per 100000 for females (no data for pancreas, liver or biliary tract) for Sweden (1983-1998). The incidence rates for Canton Vaud in Switzerland (1986-1997) provided by Levi et al. (2000) are 2.05 per 100000 for males and 2.17 per 100000 for females (no data for pancreas, liver or biliary tract; Fig. 3b). The corresponding incidence data for Austria (World standard population; all gastroenteropancreatic sites) were 2.36 per 100000 per year for females and 2.51 per 100000 for males (Fig. 3b). In our prospective study, more males than females had GEP-NETs (1.08:1). This difference is more pronounced in the USA (1.2:1, Yao et al. 2008) and in Japan (2:1, Ito et al. 2010).

\section{WHO classification: ENETS staging and grading}

Using the WHO criteria (Solcia et al. 2000), 175 $(61.4 \%)$ tumours were classified as well differentiated benign $(131,46.0 \%$; WHO 1a) or uncertain (44, $15.4 \%$; WHO $1 \mathrm{~b})$; a further $91(31.9 \%)$ were well differentiated (WHO 2) and $19(6.7 \%)$ poorly differentiated (WHO 3) neuroendocrine carcinoma.

Using the ENETS staging criteria (Rindi et al. 2006, 2007), $40.9 \%$ of tumours were stage I and $23.8 \%$ were stage II. ENETS stage III (IIIB) is defined as any T (except the location appendix), lymph node positive, but distant metastasis negative, and $11.0 \%$ fulfilled these criteria. In $23.8 \%$, stage IV was diagnosed, meaning that distant metastases were diagnosed at the time of initial diagnosis.

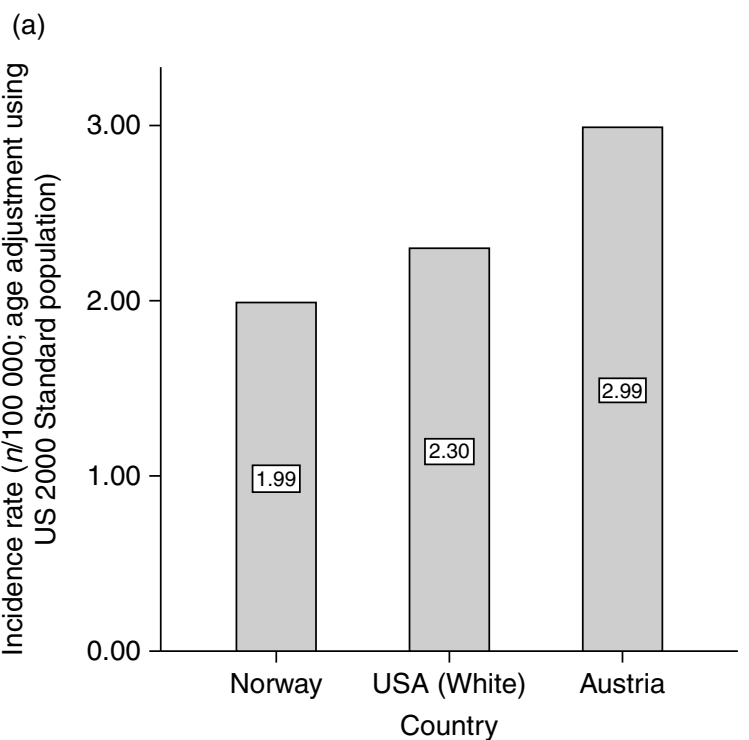

(b)

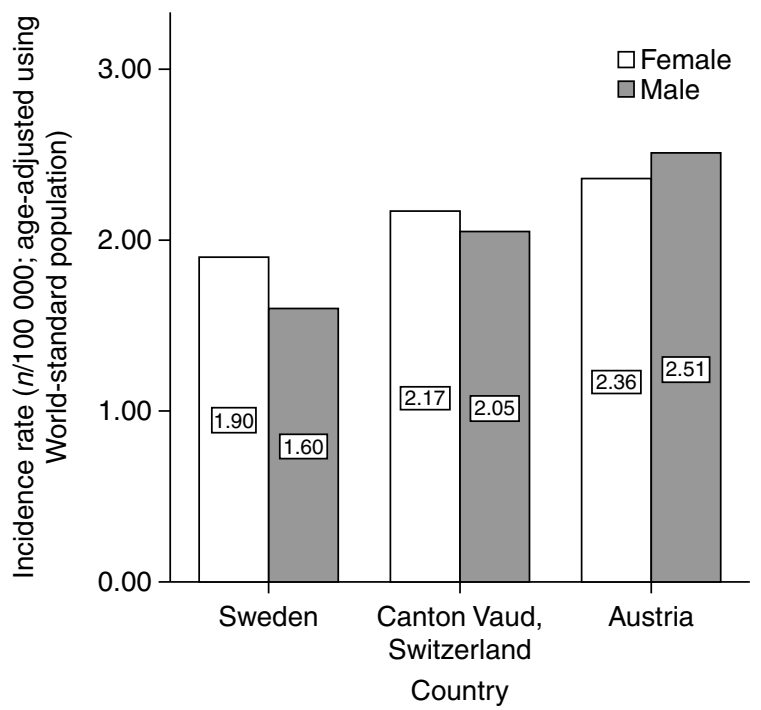

Figure 3 (a) Incidence rates of gastroenteropancreatic neuroendocrine tumours ( $n / 100000$ per year); age-adjusted using the 2000 US standard population comparing the recent data with Norway and the USA (Hauso et al. 2008).

(b) Incidence rates of gastroenteropancreatic neuroendocrine tumours (n/100 000 per year); age-adjusted using the World standard population comparing the recent data with Sweden (Hemminki \& Li 2001) and Switzerland (Levi et al. 2000).

To our knowledge, the report by Levi et al. (2000) is the only study distinguishing benign and malignant NETs on the basis of local infiltration and/or existence of metastasis: the incidence for benign NETs was 1.34 per 100000 and that for malignant tumours was 0.72 , the proportion of benign to malignant tumours being very similar to the data presented here (benign 1.15 per 100000 ; uncertain 0.43 ; malignant 0.81 ). 
Patients with malignant tumours were significantly older than patients with benign NETs, which raise the question of whether benign lesions transform into malignant tumours and therefore may be considered lesions with latent or low-grade malignancy. If this concept is accepted, the issue of early diagnosis and treatment becomes of critical clinical relevance.

In the present study, $1.49 \%$ of all malignant digestive tumours were classified as NETs. GEPNETs are diagnosed less frequently than adenocarcinomas of the digestive tract. Among malignant tumours, GEP-NETs constitute $<2 \%$ of all gastrointestinal malignancies (Maroun et al. 2006).

It has been widely discussed and long generally accepted that no histological grading system effectively predicts the behaviour of well differentiated endocrine tumours (Rindi et al. 2006). However, a grading system has been introduced by ENETS (Rindi et al. 2006, 2007) partly adopting the WHO criteria. In our study, G3 tumours were diagnosed only in the stomach, colon, rectum and pancreas.

\section{Location}

\section{Stomach}

The present study reveals the stomach as the preferential location (22.8\% of all GEP-NETs) as suspected by Kloppel \& Clemens (1996) and Kloppel et al. (2007). This is in contrast to other studies (Modlin et al. 2003, Maggard et al. 2004, Helland et al. 2006, LombardBohas et al. 2009) describing NETs of the small intestine most frequently. Reviewing clinical singlecentre studies, gastric NETs are recorded as 3.1-13.8\% (Li et al. 2008). NETs of the stomach comprise $4.1 \%$ of all gastrointestinal NETs in the late SEER (Modlin et al. 2003) and $8 \%$ in the population-based study from western Norway (Helland et al. 2006). An explanation for the increase may be the large number and liberal use of endoscopic diagnostic procedures, with biopsies routinely taken from all, even small, gastric lesions.

By definition (Kloppel et al. 2004), 67.7\% of the gastric lesions in our study were classified as NETs with benign behaviour, $12.3 \%$ as uncertain and $20.0 \%$ as malignant. The incidence of malignant NETs in the stomach was low, calculated as 0.08 per 100000 . In contrast to other studies (Rindi et al. 1996), a higher proportion of benign tumours in women with stomach NETs was not documented in our study.

\section{Duodenum/small intestine}

Even when small intestine (15.4\%) and duodenal $(5.6 \%)$ NETs are added together $(21.0 \%)$, the stomach remains the most frequent location in our study. Previously, duodenal NETs accounted for $3.8 \%$ of all gastrointestinal NETs and were integrated in the group of small bowel NETs in older series (total 25.2\%; Modlin et al. 2003). It appears unjustified to sum NETs of the small intestine and duodenum since our data document that small intestine NETs are predominantly malignant $(86.4 \%)$ and duodenal NETs are more often benign (62.5\%). The incidence of malignant NETs in the duodenum was very low at 0.02 per 100000 , whereas the incidence of malignant NETs in the ileum/jejunum was 0.29 per 100000 .

\section{Pancreas}

In our study, the overall annual incidence of PNETs was 0.25 per 100000 , subdividing into benign, uncertain and malignant at 0.02, 0.04 and 0.19 per 100000 respectively. The annual incidence of PNETs was reviewed recently and was described as $<0.4$ cases per 100000 (Halfdanarson et al. 2008), accounting for only $1-3 \%$ of all pancreatic neoplasms.

\section{Appendix}

Patients with appendiceal NETs were significantly younger (median 38.0 years) than patients with tumours in all other sites: children and adults $\leq 20$ years may be affected, as found in $5(8.9 \%)$ of 56 patients. One may speculate that appendiceal NETs are diagnosed incidentally during appendectomy because appendicitis is diagnosed more often in younger patients. Overall appendiceal NETs accounted for $20.7 \%$ of all gastrointestinal NETs, the majority $(62.7 \%)$ being classified as benign, $27.1 \%$ as uncertain and $10.2 \%$ as malignant. The incidence of malignant NETs in this location was 0.08 per 100000 population. Although the frequency of appendiceal NETs has decreased over time (Modlin et al. 2003), NETs are the most frequent type of tumour in the appendix and can be expected in 1 of 200 appendectomies (Godwin 1975). They rarely reach clinical significance.

\section{Colon and rectum}

In the analysis by Modlin \& Sandor (1997) and Modlin et al. (2003), 1481 (21.2\%) of 6996 digestive NETs were localised in the rectum, comprising $1-2 \%$ of all rectal tumours, and $938(21.2 \%)$ were in the colon. In the current study, $40(14 \%)$ of 285 GEP-NETs were located in the rectum and $20(7 \%)$ in the colon. Although the majority of those in the rectum were classified as benign ( 26 of $40,65 \%$ ), the incidence of malignant rectal NETs being only 0.03 per 100000 
population, $14(70 \%)$ of 20 colonic NETs were classified as neuroendocrine carcinoma (well differentiated $10,71 \%$; poorly differentiated $4,29 \%$; 0.13 per 100000 ).

\section{Conclusion}

Standard cancer registries do not reflect the real incidence because benign tumours are omitted from many national registries. Using strict pathohistological/immunohistochemical criteria, the incidence of benign and malignant gastrointestinal NETs has been demonstrated for the first time in a middle European country. As presumed, NETs are most frequently located in the stomach followed by the sites appendix, small intestine, rectum, pancreas and colon. Subdividing them into groups as recommended by the WHO, $46 \%$ of NETs were classified as 'benign', $15 \%$ as showing 'uncertain' biological behaviour and $39 \%$ as showing 'malignant' behaviour. Using the ENETS TNM classification, a minority of gastrointestinal NETs were classified as stage IIIB (N positive 11.0\%; regional disease) and stage IV ( $\mathrm{N}$ and/or $\mathrm{M}$ positive $23.8 \%$; distant disease) at the time of diagnosis. In the ENETS grading system, the majority of NETs were G1 (59.7\%) and G2 (31.2\%) independent of their staging. G3 tumours are very rare $(9.1 \%)$.

\section{Declaration of interest}

The authors declare that there is no conflict of interest that could be perceived as prejudicing the impartiality of the research reported.

\section{Funding}

This research did not receive any specific grant from any funding agency in the public, commercial or notfor-profit sector.

\section{Author contribution statement}

This manuscript is part of M B Niederle's doctoral thesis 'Neuroendocrine tumours of the digestive tract in Austria' (N090 Endocrinology and Metabolism) for obtaining the academic title Doctor of Medical Science at the Medical University of Vienna.

\section{Acknowledgements}

The authors wish to thank Bettina Haidbauer for running the study and secretariat and the members of the Austrian Society of Clinical Pathology for supporting this national study.

\section{References}

Berge T \& Linell F 1976 Carcinoid tumours. Frequency in a defined population during a 12-year period. Acta Pathologica, Microbiologica, et Immunologica Scandinavica 84 322-330. (doi:10.1111/j.1699-0463. 1976.tb00122.x)

Crocetti E, Buiatti E \& Amorosi A 1997 Epidemiology of carcinoid tumours in central Italy. European Journal of Epidemiology 13 357-359. (doi:10.1023/ A:1007334217762)

Doll R, Payne P \& Waterhouse J Eds 1966 Cancer Incidence in Five Continents: A Technical Report. Berlin, Germany: Springer-Verlag (for UICC).

Gatta G, Ciccolallo L, Kunkler I, Capocaccia R, Berrino F, Coleman MP, De Angelis R, Faivre J, Lutz JM, Martinez C et al. 2006 Survival from rare cancer in adults: a population-based study. Lancet Oncology 7 132-140. (doi:10.1016/S1470-2045(05)70471-X)

Godwin JD II 1975 Carcinoid tumors. An analysis of 2,837 cases. Cancer 36 560-569. (doi:10.1002/1097-0142 (197508)36:2<560::AID-CNCR2820360235>3.0. $\mathrm{CO} ; 2-4)$

Halfdanarson TR, Rubin J, Farnell MB, Grant CS \& Petersen GM 2008 Pancreatic endocrine neoplasms: epidemiology and prognosis of pancreatic endocrine tumors. Endocrine-Related Cancer 15 409-427. (doi:10. 1677/ERC-07-0221)

Hauso O, Gustafsson BI, Kidd M, Waldum HL, Drozdov I, Chan AK \& Modlin IM 2008 Neuroendocrine tumor epidemiology: contrasting Norway and North America. Cancer 113 2655-2664. (doi:10.1002/cncr.23883)

Helland SK, Prosch AM \& Viste A 2006 Carcinoid tumours in the gastrointestinal tract - a population-based study from Western Norway. Scandinavian Journal of Surgery 95 158-161.

Hemminki K \& Li X 2001 Incidence trends and risk factors of carcinoid tumors: a nationwide epidemiologic study from Sweden. Cancer 92 2204-2210. (doi:10.1002/1097-0142 (20011015)92:8 < 2204::AID-CNCR1564>3.0.CO;2-R)

Ito T, Sasano H, Tanaka M, Osamura RY, Sasaki I, Kimura W, Takano K, Obara T, Ishibashi M, Nakao K et al. 2010 Epidemiological study of gastroenteropancreatic neuroendocrine tumors in Japan. Journal of Gastroenterology 45 234-243. (doi:10.1007/s00535-009-0194-8)

Klein RJ \& Schoenborn CA 2001 Age adjustment using the 2000 projected U.S. population. Healthy People 2010 Statistical Notes 20 1-10.

Klimstra DS, Modlin IR, Adsay NV, Chetty R, Deshpande V, Gonen M, Jensen RT, Kidd M, Kulke M, Lloyd R et al. 2010 Pathology reporting of neuroendocrine tumors: application of the delphic consensus process to the development of a minimum pathology data set. American Journal of Surgical Pathology 34 300-313. (doi:10.1097/ PAS.0b013e3181ce1447)

Kloppel G 2007a Neuroendocrine tumors (2007): Oberndorfer's legacy. Virchows Archiv 451 (Supplement 1) S1. (doi:10.1007/s00428-007-0464-x) 
Kloppel G 2007b Tumour biology and histopathology of neuroendocrine tumours. Best Practice and Research. Clinical Endocrinology and Metabolism 21 15-31. (doi:10.1016/j.beem.2007.01.004)

Kloppel G \& Clemens A 1996 The biological relevance of gastric neuroendocrine tumors. Yale Journal of Biology and Medicine 69 69-74.

Kloppel G, Perren A \& Heitz PU 2004 The gastroenteropancreatic neuroendocrine cell system and its tumors: the WHO classification. Annals of the New York Academy of Sciences 1014 13-27. (doi:10.1196/annals.1294.002)

Kloppel G, Rindi G, Anlauf M, Perren A \& Komminoth P 2007 Site-specific biology and pathology of gastroenteropancreatic neuroendocrine tumors. Virchows Archiv 451 (Supplement 1) S9-S27. (doi:10.1007/ s00428-007-0461-0)

Kloppel G, Couvelard A, Perren A, Komminoth P, McNicol AM, Nilsson O, Scarpa A, Scoazec JY, Wiedenmann B, Papotti M et al. 2009 ENETS Consensus Guidelines for the Standards of Care in Neuroendocrine Tumors: towards a standardized approach to the diagnosis of gastroenteropancreatic neuroendocrine tumors and their prognostic stratification. Neuroendocrinology 90 162-166. (doi:10. 1159/000182196)

Levi F, Te VC, Randimbison L, Rindi G \& La Vecchia C 2000 Epidemiology of carcinoid neoplasms in Vaud, Switzerland, 1974-97. British Journal of Cancer $\mathbf{8 3}$ 952-955. (doi:10.1054/bjoc.2000.1394)

Li AF, Hsu CY, Li A, Tai LC, Liang WY, Li WY, Tsay SH \& Chen JY 2008 A 35-year retrospective study of carcinoid tumors in Taiwan: differences in distribution with a high probability of associated second primary malignancies. Cancer 112 274-283. (doi:10.1002/cncr.23159)

Lombard-Bohas C, Mitry E, O'Toole D, Louvet C, Pillon D, Cadiot G, Borson-Chazot F, Aparicio T, Ducreux M, Lecomte T et al. 2009 Thirteen-month registration of patients with gastroenteropancreatic endocrine tumours in France. Neuroendocrinology 89 217-222. (doi:10.1159/ 000151562)

Maggard MA, O'Connell JB \& Ko CY 2004 Updated population-based review of carcinoid tumors. Annals of Surgery 240 117-122. (doi:10.1097/01.sla.0000129342. 67174.67)

Maroun J, Kocha W, Kvols L, Bjarnason G, Chen E, Germond C, Hanna S, Poitras P, Rayson D, Reid R et al. 2006 Guidelines for the diagnosis and management of carcinoid tumours. Part 1: the gastrointestinal tract. A statement from a Canadian National Carcinoid Expert Group. Current Oncology 13 67-76.

Modlin IM \& Sandor A 1997 An analysis of 8305 cases of carcinoid tumors. Cancer 79 813-829. (doi:10.1002/(SICI)1097-0142(19970215)79: $4<813::$ AID-CNCR19>3.0.CO;2-2)
Modlin IM, Lye KD \& Kidd M 2003 A 5-decade analysis of 13,715 carcinoid tumors. Cancer 97 934-959. (doi:10. 1002/cncr.11105)

Modlin IM, Shapiro MD, Kidd M \& Eick G 2007 Siegfried oberndorfer and the evolution of carcinoid disease. Archives of Surgery 142 187-197. (doi:10.1001/archsurg. 142.2.187)

Newton JN, Swerdlow AJ, dos Santos Silva IM, Vessey MP, Grahame-Smith DG, Primatesta P \& Reynolds DJ 1994 The epidemiology of carcinoid tumours in England and Scotland. British Journal of Cancer 70 939-942. (doi:10. 1038/bjc.1994.424)

Parkin DM, Whelan SL, Ferlay J, Teppo L \& Thomas DB Eds 2003 Cancer Incidence in Five Continents, vol VIII. Lyon, France: IARC Scientific Publications.

Rindi G, Bordi C, Rappel S, La Rosa S, Stolte M \& Solcia E 1996 Gastric carcinoids and neuroendocrine carcinomas: pathogenesis, pathology, and behavior. World Journal of Surgery 20 168-172. (doi:10.1007/s002689900026)

Rindi G, Kloppel G, Alhman H, Caplin M, Couvelard A, de Herder WW, Erikssson B, Falchetti A, Falconi M, Komminoth P et al. 2006 TNM staging of foregut (neuro)endocrine tumors: a consensus proposal including a grading system. Virchows Archiv 449 395-401. (doi:10. 1007/s00428-006-0250-1)

Rindi G, Kloppel G, Couvelard A, Komminoth P, Korner M, Lopes JM, McNicol AM, Nilsson O, Perren A, Scarpa A et al. 2007 TNM staging of midgut and hindgut (neuro) endocrine tumors: a consensus proposal including a grading system. Virchows Archiv 451 757-762. (doi:10. 1007/s00428-007-0452-1)

Segi M, Fujisaku S, Kurihara M, Narai Y \& Sasajima K 1960 The age-adjusted death rates for malignant neoplasms in some selected sites in 23 countries in 1954-1955 and their geographical correlation. Tohoku Journal of Experimental Medicine 72 91-103. (doi:10.1620/tjem.72.91)

Soga J 1997 Carcinoids of the small intestine: a statistical evaluation of 1102 cases collected from the literature. Journal of Experimental \& Clinical Cancer Research 16 353-363.

Solcia E, Klöppel G \& Sobin LH 2000 Histological Typing of Endocrine Tumours, 2nd edn. World Health Organization International Histological Classification of Tumours. Berlin: Springer.

Taal BG \& Visser O 2004 Epidemiology of neuroendocrine tumours. Neuroendocrinology 80 (Supplement 1) 3-7. (doi:10.1159/000080731)

Yao JC, Hassan M, Phan A, Dagohoy C, Leary C, Mares JE, Abdalla EK, Fleming JB, Vauthey JN, Rashid A et al. 2008 One hundred years after "carcinoid": epidemiology of and prognostic factors for neuroendocrine tumors in 35,825 cases in the United States. Journal of Clinical Oncology 26 3063-3072. (doi:10.1200/JCO.2007.15.4377) 